\title{
The Solvability and Numerical Simulation for the Elastic Beam Problems with Nonlinear Boundary Conditions
}

\author{
Qun Gao \\ School of Business, Shandong University of Technology, Zibo 255049, China \\ Correspondence should be addressed to Qun Gao; qgao2014@163.com \\ Received 24 April 2014; Accepted 12 May 2014; Published 22 May 2014 \\ Academic Editor: Juntao Sun \\ Copyright (C) 2014 Qun Gao. This is an open access article distributed under the Creative Commons Attribution License, which \\ permits unrestricted use, distribution, and reproduction in any medium, provided the original work is properly cited. \\ We study the existence of multiple solutions for a fourth-order nonlinear boundary value problem. We give some new criteria for \\ guaranteeing that the fourth-order elastic beam equation with a perturbed term has at least three solutions. The proof is based on \\ some three critical points theorems of B. Ricceri. Furthermore, numerical simulations are also presented.
}

\section{Introduction}

In this paper, we consider the following elastic beam equation with nonlinear boundary conditions:

$$
\begin{aligned}
u^{(4)} & =\lambda f(t, u)+\mu g(t, u), \quad 0<t<1, \\
u(0) & =u^{\prime}(0)=0, \\
u^{\prime \prime}(1) & =0, \quad u^{\prime \prime \prime}(1)=h(u(1)),
\end{aligned}
$$

where $\lambda, \mu$ are two positive parameters, $f, g$ are two $L^{1}$ Carathéodory's functions, and $h \in C(\mathbb{R})$ is real function. This kind of problem arises in the study of deflections of elastic beams on nonlinear elastic foundations. The problem has the following physical description: a thin flexible elastic beam of length 1 is clamped at its left end $t=0$ and resting on an elastic device at its right end $t=1$, which is given by $h$. Then, the problem models the static equilibrium of the beam under a load, along its length, characterized by $f$ and $h$. The derivation of the model can be found in $[1,2]$.

In recent years, fourth-order boundary value problems modeling bending equilibria of elastic beams have been extensively studied by many researchers. We refer the reader to [1-8]. Some of them are concerned with nonlinear equations with null boundary conditions; see [3-6]. Others are concerned with nonlinear equations with nonzero or nonlinear boundary conditions, which can model beams resting on elastic bearings located in their extremities; see, for instance, $[1,2,7,8]$ and the references therein.

Let us briefly comment the known results related to problem (1). In [2], Ma studied the existence of positive solutions for problem (1) with $\lambda=1$ and $\mu=0$ using mountain pass theorem. Later, Yang et al. [8] considered the existence of two solutions for problem (1) with $\mu=0$, which are generated by the function $h$. Very recently, Li and Sun [9] obtained the existence of infinitely many solutions for problem (1) with $\lambda=1$ and $\mu=0$ by using the fountain theorem.

Motivated by the above works, in the present paper, we establish some existence results of three solutions for problem (1) under rather different assumptions on functions $f, h$, and $g$. We require that $f$ satisfies the asymptotically linear or sublinear conditions at infinity on $u$. The proof is based on some three critical points theorems due to Ricceri. It is worth noting that these critical points theorems have been extensively applied in the study of the existence of solutions for nonlinear differential equations; see [10-13].

The remainder of this paper is organized as follows. In Section 2, some preliminary results are presented. In Section 3, we give the proofs of our main results. In addition, some numerical simulations are presented at the end of the paper. 


\section{Variational Setting and Preliminaries}

If $X$ is a real Banach space, denote by $\mathscr{W}_{X}$ the class of all functionals $\Phi: X \rightarrow \mathbb{R}$ possessing the following property: if $\left\{u_{n}\right\}$ is a sequence in $X$ converging weakly to $u \in X$ and $\liminf _{n \rightarrow \infty} \Phi\left(u_{n}\right) \leq \Phi(u)$, then $\left\{u_{n}\right\}$ has a subsequence converging strongly to $u$.

For example, if $X$ is uniformly convex and $g:[0,+\infty) \rightarrow$ $\mathbb{R}$ is a continuous, strictly increasing function, then, by a classical result, the functional $u \rightarrow g(\|u\|)$ belongs to the class $\mathscr{W}_{X}$.

Theorem 1 (see [14]). Let $X$ be a separable and reflexive real Banach space; let $\Phi: X \rightarrow \mathbb{R}$ be a coercive, sequentially weakly lower semicontinuous $C^{1}$ functional, belonging to $\mathscr{W}_{X}$, bounded on each bounded subset of $X$ and whose derivative admits a continuous inverse on $X^{*} ; J: X \rightarrow \mathbb{R}$ a $C^{1}$ functional with compact derivative. Assume that $\Phi$ has a strict local minimum $u_{0}$ with $\Phi\left(u_{0}\right)=J\left(u_{0}\right)=0$. Finally, setting

$$
\begin{aligned}
& \alpha=\max \left\{0, \limsup _{\|u\| \rightarrow+\infty} \frac{J(u)}{\Phi(u)}, \limsup _{u \rightarrow u_{0}} \frac{J(u)}{\Phi(u)}\right\}, \\
& \beta=\sup _{u \in \Phi^{-1}((0,+\infty))} \frac{J(u)}{\Phi(u)},
\end{aligned}
$$

assume that $\alpha<\beta$.

Then, for each compact interval $[a, b] \subset(1 / \beta, 1 / \alpha)$ (with the conventions $1 / 0=+\infty, 1 /+\infty=0)$, there exists $R>0$ with the following property: for every $\lambda \in[a, b]$ and every $C^{1}$ functional $\Psi: X \rightarrow \mathbb{R}$ with compact derivative, there exists $\delta>0$ such that, for each $\mu \in[0, \delta]$,

$$
\Phi^{\prime}(x)=\lambda J^{\prime}(x)+\mu \Psi^{\prime}(x)
$$

has at least three solutions in $X$ whose norms are less than $R$.

The following two results of Ricceri guarantee the existence of three solutions for a given equation.

Theorem 2 (see [15]). Let $X$ be a reflexive real Banach space; $I \subseteq \mathbb{R}$ an interval; let $\Phi: X \rightarrow \mathbb{R}$ be a sequentially weakly lower semicontinuous $C^{1}$ functional, bounded on each bounded subset of $X$, whose derivative admits a continuous inverse on $X^{*} ;-J: X \rightarrow \mathbb{R} a C^{1}$ functional with compact derivative. Assume that

$$
\lim _{\|x\| \rightarrow+\infty}(\Phi(x)-\lambda J(x))=+\infty
$$

for all $\lambda \in I$, and that there exists $\rho \in \mathbb{R}$ such that

$$
\begin{aligned}
\sup _{\lambda \in I} \inf _{x \in X}(\Phi(x)+\lambda(\rho-J(x))) & \\
& <\inf _{x \in X} \sup _{\lambda \in I}(\Phi(x)+\lambda(\rho-J(x))) .
\end{aligned}
$$

Then, there exist a nonempty open set $A \subseteq I$ and a positive number $R$ with the following property: for every $\lambda \in A$ and every $C^{1}$ functional $-\Psi: X \rightarrow \mathbb{R}$ with compact derivative, there exists $\delta>0$ such that, for each $\mu \in[0, \delta]$,

$$
\Phi^{\prime}(x)-\lambda J^{\prime}(x)-\mu \Psi^{\prime}(x)=0
$$

has at least three solutions in $X$ whose norms are less than $R$.
Proposition 3 (see [16]). Let $X$ be a nonempty set and $\Phi, J$ two real functions on $X$. Assume that there are $r>0$ and $x_{0}$, $x_{1} \in X$ such that

$$
\begin{gathered}
\Phi\left(x_{0}\right)=J\left(x_{0}\right)=0, \quad \Phi\left(x_{1}\right)>r, \\
\sup _{x \in \Phi^{-1}((-\infty, r])} J(x)<r \frac{J\left(x_{1}\right)}{\Phi\left(x_{1}\right)} .
\end{gathered}
$$

Then, for each $\rho$ satisfying

$$
\sup _{x \in \Phi^{-1}((-\infty, r])} J(x)<\rho<r \frac{J\left(x_{1}\right)}{\Phi\left(x_{1}\right)},
$$

one has

$$
\begin{aligned}
\sup _{\lambda \geq 0} \inf _{x \in X}(\Phi(x)+\lambda(\rho-J(x))) & \\
& <\inf _{x \in X} \sup _{\lambda \geq 0}(\Phi(x)+\lambda(\rho-J(x))) .
\end{aligned}
$$

Now, we begin describing the variational formulation of problem (1), which is based on the function space

$$
E=\left\{u \in H^{2}(0,1) ; u(0)=u^{\prime}(0)=0\right\},
$$

where $H^{2}(0,1)$ is the Sobolev space of all functions $u$ : $[0,1] \rightarrow \mathbb{R}$ such that $u$ and its distributional derivative $u^{\prime}$ are absolutely continuous and $u^{\prime \prime}$ belongs to $L^{2}([0,1])$. Then, $E$ is a Hilbert space equipped with the inner product and norm

$$
\langle u, v\rangle=\int_{0}^{1} u^{\prime \prime}(t) v^{\prime \prime}(t) d t, \quad\|u\|=\left\|u^{\prime \prime}\right\|_{2},
$$

where $\|\cdot\|_{p}$ denotes the standard $L^{p}$-norm. In addition, $E$ is compactly embedded in the spaces $L^{2}([0,1])$ and $C([0,1])$, and, therefore, there exist immersion constants $S_{2}, \bar{S}>0$, such that

$$
\|u\|_{2} \leq S_{2}\|u\|, \quad\|u\|_{\infty} \leq \bar{S}\|u\|
$$

We recall that $f:[0,1] \times \mathbb{R} \rightarrow \mathbb{R}$ is an $L^{1}$-Carathéodory function if

(a) the mapping $t \mapsto f(t, u)$ is measurable for every $u \in$ $\mathbb{R}$;

(b) the mapping $u \mapsto f(t, u)$ is continuous for almost every $t \in[0,1]$;

(c) for every $\rho>0$ there exists a function $l_{\rho} \in L^{1}([0,1])$ such that

$$
\sup _{|u| \leq \rho}|f(t, u)| \leq l_{\rho}(t)
$$

for almost every $t \in[0,1]$.

Definition 4. One says that a function $u \in E$ is a weak solution of problem (1) if

$$
\begin{aligned}
& \int_{0}^{1}\left(u^{\prime \prime}(t), v^{\prime \prime}(t)\right) d t+h(u(1)) v(1) \\
& \quad=\lambda \int_{0}^{1} f(t, u) v d t+\mu \int_{0}^{1} g(t, u) v d t
\end{aligned}
$$

holds for any $v \in E$. 
In order to study problem (1), we will use the functionals $\Phi, J$, and $\Psi: E \rightarrow \mathbb{R}$ defined by putting

$$
\begin{aligned}
& \Phi(u)=\frac{1}{2}\|u\|^{2}+\int_{0}^{u(1)} h(s) d s=\phi(u)+\psi(u), \\
& J(u):=\int_{0}^{1} F(t, u) d t, \quad \Psi(u):=\int_{0}^{1} G(t, u) d t,
\end{aligned}
$$

respectively, for every $u \in E$, where,

$$
\begin{aligned}
\phi(u) & =\frac{1}{2}\|u\|^{2}, \quad \psi(u)=\int_{0}^{u(1)} h(t) d t, \\
F(t, u) & =\int_{0}^{u} f(t, s) d s, \quad G(t, u)=\int_{0}^{u} g(t, s) d s .
\end{aligned}
$$

By the continuity of $h$, we get that functional $\Phi$ is a continuous Gâteaux differential functional whose Gâteaux derivative is the functional $\Phi^{\prime}(u)$, given by

$$
\left\langle\Phi^{\prime}(u), v\right\rangle=\int_{0}^{1}\left(u^{\prime \prime}(t), v^{\prime \prime}(t)\right) d t+h(u(1)) v(1)
$$

for any $u, v \in E$. Since $f, g$ are two $L^{1}$-Carathéodory functions, it implies that $J$ and $\Psi$ are well defined and continuously Gâteaux differentiable in E. More precisely, their Gâteaux derivatives are

$$
\begin{aligned}
& \left\langle J^{\prime}(u), v\right\rangle=\int_{0}^{1} f(t, u) v d t, \\
& \left\langle\Psi^{\prime}(u), v\right\rangle=\int_{0}^{1} g(t, u) v d t,
\end{aligned}
$$

respectively, for every $u, v \in E$.

Lemma 5. Assume that the following condition holds:

$\left(\mathrm{H}_{1}\right) h(s)$ are nondecreasing on $s \in \mathbb{R}$ and $h(s) s \geq 0$, for any $s \in \mathbb{R}$.

Then, $\Phi$ is sequentially weakly lower semicontinuous, bounded on each bounded subset of $E$, and its derivative admits a continuous inverse.

Proof. Obviously, $\phi$ is weakly lower semicontinuous in $E$. Therefore, it suffices to show that $\psi$ is weakly continuous in $E$. In fact, if $\left\{u_{n}\right\} \subset E$ and $u_{n} \rightarrow u$ in $E,\left\{u_{n}\right\}$ converges uniformly to $u$ on $[0,1]$. Then, there exists $C_{1}>0$ such that

$$
\left\|u_{n}\right\|_{\infty} \leq C_{1}, \quad \forall n \in \mathbb{N} .
$$

Therefore, we have

$$
\begin{aligned}
\left|\psi\left(u_{n}\right)-\psi(u)\right| & =\left|\int_{0}^{u_{n}(1)} h(t) d t-\int_{0}^{u(1)} h(t) d t\right| \\
& =\left|\int_{u(1)}^{u_{n}(1)} h(t) d t\right| \\
& \leq C_{2}\left\|u_{n}-u\right\|_{\infty} \longrightarrow 0,
\end{aligned}
$$

where $C_{2}=\max _{|t| \leq C_{1}}|h(t)|$. Thus, $\psi$ is weakly continuous. Therefore, $\Phi$ is weakly lower semicontinuous in $E$.

Moreover, let $\Omega$ be a bounded subset of $E$; that is, there exists a constant $a>0$ such that $\|u\| \leq a$ for $u \in \Omega$. Then,

$$
\begin{aligned}
|\Phi(u)| & =\left|\frac{1}{2}\|u\|^{2}+\int_{0}^{u(1)} h(t) d t\right| \\
& \leq \frac{1}{2} a^{2}+\max _{|t| \leq \bar{S} a}|h(t)| \cdot\|u\|_{\infty} \\
& \leq \frac{1}{2} a^{2}+\bar{S} \max _{|t| \leq \bar{S} a}|h(t)| \cdot\|u\| \\
& \leq \frac{1}{2} a^{2}+\bar{S} a \max _{|t| \leq \bar{S} a}|h(t)| .
\end{aligned}
$$

Hence, $\Phi$ is bounded on each bounded subset of $E$.

Next, we show that $\Phi^{\prime}$ admits a continuous inverse. For any $u \in E \backslash\{0\}$, by $\left(\mathrm{H}_{1}\right)$, we have

$$
\begin{aligned}
\left\langle\Phi^{\prime}(u), u\right\rangle & =\int_{0}^{1}\left(u^{\prime \prime}(t), u^{\prime \prime}(t)\right) d t+h(u(1)) u(1) \\
& \geq \int_{0}^{1}\left(u^{\prime \prime}(t), u^{\prime \prime}(t)\right) d t=\|u\|^{2} .
\end{aligned}
$$

So, $\lim _{\|u\| \rightarrow+\infty}\left\langle\Phi^{\prime}(u), u\right\rangle /\|u\|=+\infty$; that is, $\Phi^{\prime}$ is coercive.

For any $u, v \in E$, by $\left(\mathrm{H}_{1}\right)$, one has

$$
\begin{aligned}
&\left\langle\Phi^{\prime}(u)-\Phi^{\prime}(v), u-v\right\rangle \\
&= \int_{0}^{1}\left(u^{\prime \prime}-v^{\prime \prime}, u^{\prime \prime}-v^{\prime \prime}\right) d t \\
&+(h(u(1))-h(v(1)))(u(1)-v(1)) \\
& \geq\|u-v\|^{2} .
\end{aligned}
$$

So, $\Phi^{\prime}$ is uniformly monotone. By [17, Theorem 26.A (d)], we have that $\left(\Phi^{\prime}\right)^{-1}$ exists and is continuous.

Remark 6. If $\left(\mathrm{H}_{1}\right)$ is replaced by the following condition:

$\left(\mathrm{H}_{1}\right)^{\prime} g(s)$ are odd and nondecreasing on $s \in \mathbb{R}$.

Then, we can obtain the same conclusion.

Lemma 7. $J$ and $\Psi$ are continuously Gateaux differentiable in $E$ and their derivatives are compact. 
Proof. It is easy to verify that $J$ and $\Psi$ are continuously Gâteaux differentiable in $E$. Now, we proof that their derivatives are compact.

For any $u, v \in E$,

$$
\left\langle J^{\prime}(u), v\right\rangle=\int_{0}^{1} f(t, u) v d t .
$$

Let $u_{n} \rightarrow u$ as $n \rightarrow \infty$ in $E$. Then, $u_{n} \rightarrow u$ in $C([0,1])$. Since $f(t, u)$ is continuous in $u$, one has $f\left(t, u_{n}\right) \rightarrow f(t, u)$ as $n \rightarrow \infty$. So $J^{\prime}\left(u_{n}\right) \rightarrow J^{\prime}(u)$ as $n \rightarrow \infty$. That is, $J^{\prime}$ is strongly continuous on $E$, which implies that $J^{\prime}$ is a compact operator by [17, Proposition 26.2]. Moreover, $J^{\prime}$ is continuous since it is strongly continuous.

Analogously, we have that $\Psi^{\prime}(u)$ is a compact operator for any $u$.

\section{Main Results}

In this section, we establish the main abstract results of this paper. Put

$$
\begin{aligned}
& \lambda_{1}=\inf \left\{\frac{\|u\|^{2}+2 \int_{0}^{u(1)} h(t) d t}{2 \int_{0}^{T} F(t, u(t)) d t}: u \in E,\right. \\
& \left.\int_{0}^{T} F(t, u(t)) d t>0\right\}, \\
& \lambda_{2}^{-1}=\max \left\{0, \limsup _{\|u\| \rightarrow+\infty} \frac{2 \int_{0}^{T} F(t, u(t)) d t}{\|u\|^{2}+2 \int_{0}^{u(1)} h(t) d t},\right. \\
& \left.\limsup _{|u| \rightarrow 0} \frac{2 \int_{0}^{T} F(t, u(t)) d t}{\|u\|^{2}+2 \int_{0}^{u(1)} h(t) d t}\right\} \text {. }
\end{aligned}
$$

Theorem 8. Suppose that $f:[0,1] \times \mathbb{R} \rightarrow \mathbb{R}$ is an $L^{1}$ Carathéodory function and $\left(H_{1}\right)\left(\right.$ or $\left.\left(H_{1}\right)^{\prime}\right)$ holds. Moreover, assume that the following conditions hold:

$\left(\mathrm{R}_{1}\right)$ there exists a constant $p>0$ such that

$$
\begin{aligned}
\max & \left\{\limsup _{u \rightarrow 0} \frac{\max _{t \in[0,1]} F(t, u)}{|u|^{2}}, \limsup _{|u| \rightarrow+\infty} \frac{\max _{t \in[0,1]} F(t, u)}{|u|^{2}}\right\} \\
& <p
\end{aligned}
$$

$\left(\mathrm{R}_{2}\right)$ there exists a function $c \in C^{2}([0,1])$ satisfying $c(0)=$ $c^{\prime}(0)=0$ and

$$
\int_{0}^{1}\left|c^{\prime \prime}\right|^{2} d t+2 \int_{0}^{c(1)} h(t) d t \neq 0,
$$

such that

$$
p \bar{S}^{2}<\frac{\int_{0}^{1} F(t, c(t)) d t}{\int_{0}^{1}\left|c^{\prime \prime}\right|^{2} d t+2 \int_{0}^{c(1)} h(t) d t}
$$

Then, for each compact interval $[a, b] \subset\left(\lambda_{1}, \lambda_{2}\right)$, there exists $R>0$ with the following property: for every $\lambda \in[a, b]$ and for any $L^{1}$-Carathéodory function $h:[0,1] \times \mathbb{R} \rightarrow \mathbb{R}$, there exists $\delta>0$ such that, for each $\mu \in[0, \delta]$, problem (1) has at least three weak solutions whose norms are less than $R$.

Proof. Obviously, $E$ is a separable and uniformly convex Banach space. By Lemmas 5 and 7 , we obtain that $\Phi$ is a continuous Gâteaux derivative, sequentially weakly lower semicontinuous, bounded on each bounded subset of $E$, and its derivative admits a continuous inverse; $J, \Psi$ are the Gâteaux derivative functionals whose derivatives are compact. It is clear that $\phi \in \mathscr{W}_{E}$ (see the beginning of Section 2). Now, we show that $\Phi \in E$. For a sequence $\left\{u_{n}\right\} \subset$ $E$, if $\left\{u_{n}\right\} \rightarrow u \in E$ and $\liminf _{n \rightarrow \infty} \Phi\left(u_{n}\right) \leq \Phi(u)$, in view of the weak continuity of $\psi$, one has $\liminf _{n \rightarrow \infty} \phi\left(u_{n}\right) \leq$ $\phi(u)$. Thus, $\left\{u_{n}\right\}$ has a subsequence converging strongly to $u$. Therefore, $\Phi \in \mathscr{W}_{E}$. Moreover, $\Phi$ is coercive and has a strict local minimum 0 with $\Phi(0)=J(0)=0$.

In view of $\left(\mathrm{R}_{1}\right)$, there exist $\tau_{1}, \tau_{2}$ with $0<\tau_{1}<\tau_{2}$ such that

$$
F(t, u) \leq p|u|^{2}
$$

for any $t \in[0,1]$ and $u$ with $|u| \in\left[0, \tau_{1}\right) \cup\left(\tau_{2},+\infty\right)$. Since $f$ is a $L^{1}$-Carathéodory function, $F(t, u)$ is bounded on $t \in[0,1]$ and $u$ with $|u| \in\left[\tau_{1}, \tau_{2}\right]$; we can choose $\rho>0$ and $\sigma>2$ in such a way that

$$
F(t, u) \leq p|u|^{2}+\rho|u|^{\sigma},
$$

for all $(t, u) \in[0,1] \times \mathbb{R}$. So, by $(12)$, one has

$$
J(u) \leq p \bar{S}^{2}\|u\|^{2}+\rho \bar{S}^{\sigma}\|u\|^{\sigma},
$$

for all $u \in E$. Hence, using $\left(\mathrm{H}_{1}\right)$ and (31), we have

$$
\limsup _{|u| \rightarrow 0} \frac{J(u)}{\Phi(u)} \leq 2 p \bar{S}^{2}
$$

Furthermore, using (29) again, for each $u \in E \backslash\{0\}$, we obtain

$$
\begin{aligned}
\frac{2 \int_{0}^{1} F(t, u(t)) d t}{\|u\|^{2}+2 \int_{0}^{u(1)} h(t) d t}= & \frac{2 \int_{|u| \leq \tau_{2}} F(t, u(t)) d t}{\|u\|^{2}+2 \int_{0}^{u(1)} h(t) d t} \\
& +\frac{2 \int_{|u|>\tau_{2}} F(t, u(t)) d t}{\|u\|^{2}+2 \int_{0}^{u(1)} h(t) d t} \\
\leq & \frac{2 \sup _{t \in[0,1],|u| \in\left[0, \tau_{2}\right]} F(t, u)}{\|u\|^{2}+2 \int_{0}^{u(1)} h(t) d t} \\
& +\frac{2 p \bar{S}^{2}\|u\|^{2}}{\|u\|^{2}+2 \int_{0}^{u(1)} h(t) d t} \\
\leq & \frac{2 \sup _{t \in[0,1],|u| \in\left[0, \tau_{2}\right]} F(t, u)}{\|u\|^{2}+2 \int_{0}^{u(1)} h(t) d t}+2 p \bar{S}^{2} .
\end{aligned}
$$


So, we get

$$
\limsup _{\|u\| \rightarrow+\infty} \frac{J(u)}{\Phi(u)} \leq 2 p \bar{S}^{2}
$$

Combining (32) with (34), one has

$$
\alpha:=\max \left\{0, \limsup _{\|u\| \rightarrow+\infty} \frac{J(u)}{\Phi(u)}, \limsup _{|u| \rightarrow 0} \frac{J(u)}{\Phi(u)}\right\} \leq 2 p \bar{S}^{2} .
$$

By $\left(\mathrm{H}_{1}\right),\left(\mathrm{R}_{2}\right)$, and (35), we have

$$
\begin{aligned}
\beta & =\sup _{u \in \Phi^{-1}((0,+\infty))} \frac{J(u)}{\Phi(u)}=\sup _{u \in E \backslash\{0\}} \frac{J(u)}{\Phi(u)} \\
& \geq \frac{2 \int_{0}^{1} F(t, c(t)) d t}{\int_{0}^{1}\left|c^{\prime \prime}\right|^{2} d t+2 \int_{0}^{c(1)} h(t) d t}>2 p \bar{S}^{2} \geq \alpha .
\end{aligned}
$$

Then, for each compact interval $[a, b] \subset\left(\lambda_{1}, \lambda_{2}\right)$, there exists $R>0$ with the following property: for every $\lambda \in[a, b]$ and for any $L^{1}$-Carathéodory function $h:[0,1] \times \mathbb{R} \rightarrow \mathbb{R}$, there exists $\delta>0$ such that, for each $\mu \in[0, \delta]$, problem (1) has at least three weak solutions whose norms are less than $R$.

Theorem 9. Suppose that $f:[0,1] \times \mathbb{R} \rightarrow \mathbb{R}$ is an $L^{1}$ Carathéodory function, $0<\bar{t}<\widehat{t}<1$, and $\left(H_{1}\right)\left(\right.$ or $\left.\left(H_{1}\right)^{\prime}\right)$ holds. Furthermore, assume that the following conditions hold:

$\left(\mathrm{M}_{1}\right)$ there exist two functions $d \in C^{2}([0, \bar{t}])$ and $e \in$ $C^{2}([\widehat{t}, 1])$ satisfying

$$
\begin{aligned}
& d(0)=d^{\prime}(0)=0, \quad d(\bar{t})=e(\widehat{t})=1, \\
& d^{\prime}(\bar{t})=e^{\prime}(\widehat{t})=0,
\end{aligned}
$$

and two positive constants $\gamma, \delta$ with $\delta>D:=$ $\gamma \bar{S} \sqrt{\int_{0}^{\bar{t}}\left|d^{\prime \prime}\right|^{2} d t+\int_{\hat{t}}^{1}\left|e^{\prime \prime}\right|^{2} d t}$ such that

$$
\max _{t \in[0,1],|u| \leq \delta} F(t, u)
$$

$$
<\frac{\int_{\bar{t}}^{\bar{t}} F(t, \gamma) d t\left[\int_{0}^{\bar{t}}\left|d^{\prime \prime}\right|^{2} d t+\int_{\hat{t}}^{1}\left|e^{\prime \prime}\right|^{2} d t\right]}{\int_{0}^{\bar{t}}\left|d^{\prime \prime}\right|^{2} d t+\int_{\hat{t}}^{1}\left|e^{\prime \prime}\right|^{2} d t+\left(2 / \gamma^{2}\right) \int_{0}^{e(1) \gamma} h(t) d t} ;
$$

\section{$\left(\mathrm{M}_{2}\right) F(t, u) \geq 0$ for all $t \in[0, \bar{t}] \cup[\widehat{t}, 1]$ and $u \in \mathbb{R} ;$}

$\left(\mathrm{M}_{3}\right)$ there exist $w, q \in L^{1}\left(0, T ; \mathbb{R}^{+}\right)$and a positive constant $\alpha \in[0,1)$ such that $|f(t, u)| \leq w(t)|u|^{\alpha}+q(t)$, for all $(t, u) \in[0,1] \times \mathbb{R}$.

Then, there exist a nonempty open set $A \subset[0,+\infty)$ and $a$ positive number $R$ with the following property: for every $\lambda \in A$ and for any $L^{1}$-Carathéodory function $h:[0,1] \times \mathbb{R} \rightarrow \mathbb{R}$, there exists $\delta>0$ such that, for each $\mu \in[0, \delta]$, problem (1) has at least three weak solutions whose norms are less than $R$.
Proof. Obviously, $\Phi(0)=J(0)=0$. For any $\lambda \geq 0$ and $u \in E$, by $\left(\mathrm{H}_{1}\right)$ and $\left(\mathrm{M}_{3}\right)$, we have

$$
\begin{aligned}
\Phi(u)-\lambda J(u) & =\frac{1}{2}\|u\|^{2}+\int_{0}^{u(1)} h(t) d t-\lambda \int_{0}^{1} F(t, u) d t \\
& \geq \frac{1}{2}\|u\|^{2}-\lambda \int_{0}^{1}\left(\frac{w(t)}{\alpha}|u|^{\alpha+1}+q(t)|u|\right) d t \\
& \geq \frac{1}{2}\|u\|^{2}-\frac{\lambda \bar{S}^{\alpha+1}}{\alpha}\|w\|_{1}\|u\|_{\alpha+1}-\lambda\|q\|_{1}\|u\| .
\end{aligned}
$$

Since $\alpha<1$, one has $\lim _{\|u\| \rightarrow+\infty}(\Phi(u)-\lambda J(u))=+\infty$ for all $\lambda \geq 0$.

For each $r>0$ and $\Phi(u) \leq r$, we have $\|u\|_{\infty} \leq \bar{S} \sqrt{2 r}$; that is,

$$
\Phi^{-1}((-\infty, r]) \subseteq\left\{u \in E: \max _{t \in[0,1]}|u(t)| \leq \bar{S} \sqrt{2 r}\right\} .
$$

Therefore,

$$
\begin{aligned}
\sup _{u \in \Phi^{-1}((-\infty, r])} J(u) & \leq \max _{|u| \leq \bar{S} \sqrt{2 r}} J(u) \\
& =\max _{|u| \leq \bar{S} \sqrt{2 r}} \int_{0}^{1} F(t, u) d t \\
& \leq \max _{t \in[0,1],|u| \leq \bar{S} \sqrt{2 r}} F(t, u) .
\end{aligned}
$$

Define the function

$$
\eta(t):= \begin{cases}d(t) \gamma, & t \in[0, \bar{t}] \\ \gamma, & t \in(\bar{t}, \hat{t}] \\ e(t) \gamma, & t \in(\widehat{t}, 1]\end{cases}
$$

From condition $\left(\mathrm{M}_{1}\right)$, it is easy to verify that $\eta \in E$ and

$$
\begin{aligned}
\Phi(\eta) & =\frac{1}{2}\|\eta\|^{2}+\int_{0}^{\eta(1)} h(t) d t \\
& =\frac{\gamma^{2}}{2}\left[\int_{0}^{\bar{t}}\left|d^{\prime \prime}\right|^{2} d t+\int_{\widehat{t}}^{1}\left|e^{\prime \prime}\right|^{2} d t\right]+\int_{0}^{e(1) \gamma} h(t) d t .
\end{aligned}
$$

Choose $r=\left(\gamma^{2} / 2\right)\left[\int_{0}^{\bar{t}}\left|d^{\prime \prime}\right|^{2} d t+\int_{\hat{t}}^{1}\left|e^{\prime \prime}\right|^{2} d t\right]$; then, using (43) and $\left(\mathrm{H}_{1}\right)$, we have $\Phi(\eta)>r$. Furthermore, using $\left(\mathrm{M}_{1}\right),\left(\mathrm{M}_{2}\right)$, (35), and (43), we have

$$
\begin{aligned}
r \frac{J(\eta)}{\Phi(\eta)} & =r \frac{\int_{0}^{1} F(t, \eta) d t}{(1 / 2)\|\eta\|^{2}+\int_{0}^{\eta(1)} h(t) d t} \\
& \geq r \frac{\int_{\bar{t}}^{\bar{t}} F(t, \gamma) d t}{\left(\gamma^{2} / 2\right)\left[\int_{0}^{\bar{t}}\left|d^{\prime \prime}\right|^{2} d t+\int_{\hat{t}}^{1}\left|e^{\prime \prime}\right|^{2} d t\right]+\int_{0}^{e(1) \gamma} h(t) d t}
\end{aligned}
$$




$$
\begin{aligned}
& =\frac{\int_{\bar{t}}^{\widehat{t}} F(t, \gamma) d t\left[\int_{0}^{\bar{t}}\left|d^{\prime \prime}\right|^{2} d t+\int_{\hat{t}}^{1}\left|e^{\prime \prime}\right|^{2} d t\right]}{\int_{0}^{\bar{t}}\left|d^{\prime \prime}\right|^{2} d t+\int_{\hat{t}}^{1}\left|e^{\prime \prime}\right|^{2} d t+\left(2 / \gamma^{2}\right) \int_{0}^{e(1) \gamma} h(t) d t} \\
& >\max _{t \in[0,1],|u| \leq \delta} F(t, u) \\
& >\max _{t \in[0,1],|u| \leq D} F(t, u) \\
& =\max _{t \in[0,1],|u| \leq \bar{S} \sqrt{2 r}} F(t, u) \geq \sup _{u \in \Phi^{-1}((-\infty, r])} J(u) .
\end{aligned}
$$

Therefore, we can fix $\rho$ such that

$$
\sup _{u \in \Phi^{-1}((-\infty, r])} J(u)<\rho<r \frac{J(\eta)}{\Phi(\eta)} .
$$

From Proposition 3, we obtain

$$
\begin{aligned}
\sup _{\lambda \geq 0} \inf _{u \in E}(\Phi(u)+\lambda(\rho-J(u))) & \\
& <\inf _{u \in E} \sup _{\lambda \geq 0}(\Phi(u)+\lambda(\rho-J(u))) .
\end{aligned}
$$

Therefore, using Theorem 2, for each compact interval $[a, b] \subset\left(\lambda_{1}, \lambda_{2}\right)$, there exists $R>0$ with the following property: for every $\lambda \in[a, b]$ and for any $L^{1}$-Carathéodory function $h:[0,1] \times \mathbb{R} \rightarrow \mathbb{R}$, there exists $\delta>0$ such that, for each $\mu \in[0, \delta], \Phi^{\prime}(u)-\lambda J^{\prime}(u)-\mu \Psi^{\prime}(u)=0$ has at least three solutions in $E$. Hence, problem (1) has at least three weak solutions whose norms are less than $R$.

Remark 10. Clearly, Theorem 9 gives the result of at least three solutions for problem (1) with $f(t, u)$ being of sublinear growth.

\section{Numerical Solutions}

In this section, we consider a numerical algorithm for the problem (1) using the finite differences method. Let $0=t_{0}<$ $t_{1}<\cdots<t_{n}=1$ be a discretization of the interval $[0,1]$ with mesh size $m=t_{i}-t_{i-1}$. Then, putting $u_{i}=u\left(t_{i}\right)$ and $f_{i}=f\left(t_{i}, u_{i}\right)$ and using central differences formula, the first equation of problem (1) becomes

$$
u_{i-2}-4 u_{i-1}+6 u_{i}-4 u_{i+1}+u_{i+2}=m^{4} f_{i}
$$

$2 \leq i \leq n-2$. Taking into account the boundary conditions of problem (1), we infer that

$$
\begin{gathered}
u_{0}=0, \quad u_{-1}=u_{1}, \quad u_{n+1}=-u_{n-1}+2 u_{n}, \\
u_{n+2}=2 m^{3} h\left(u_{n}\right)+u_{n-2}-4 u_{n-1}+4 u_{n} .
\end{gathered}
$$

Then, we can compute $u_{1}, \ldots, u_{n}$ by solving the nonlinear system $A U=B$, with

$$
\begin{aligned}
& A=\left(\begin{array}{ccccccccccc}
7 & -4 & 1 & & & & & & & & \\
-4 & 6 & -4 & 1 & & & & & & & \\
1 & -4 & 6 & -4 & 6 & & & & & & \\
& & & & & \ddots & & & & & \\
& & & & & & 1 & -4 & 6 & -4 & 1 \\
& & & & & & & & -4 & 5 & -2 \\
& & & & & & & 2 & -4 & 2
\end{array}\right), \\
& U=\left(\begin{array}{c}
u_{1} \\
u_{2} \\
\vdots \\
u_{n-1} \\
u_{n}
\end{array}\right), \quad B=\left(\begin{array}{c}
m^{4} f_{1} \\
\vdots \\
m^{4} f_{n-1} \\
m^{4} f_{n}-2 m^{3} g\left(u_{n}\right)
\end{array}\right)
\end{aligned}
$$

Since the system is nonlinear, we use a linearization through an initial approximation $U_{0}$ and iterative methods for solving it. The quantities $f_{i}$ are updated after each iteration.

Let $\mu=0$ and fix $\lambda>0$. Choose a $f$ satisfying all conditions. Using $u(t)=t$ as initial approximation and mesh size $m=0.1$, it is easy to obtain the approximate solution with Error $:=\max \left|u_{i}-u\left(t_{i}\right)\right|$ by the Gauss-Seidel method.

\section{Conflict of Interests}

The author declares that there is no conflict of interests regarding the publication of this paper.

\section{Acknowledgment}

Q. Gao was supported by the Humanities and Social Sciences Foundation of the Ministry of Education in China (Grant no. 13YJAZH112).

\section{References}

[1] E. Alves, T. F. Ma, and M. L. Pelicer, "Monotone positive solutions for a fourth order equation with nonlinear boundary conditions," Nonlinear Analysis, Theory, Methods and Applications, vol. 71, no. 9, pp. 3834-3841, 2009.

[2] T.F. Ma, "Positive solutions for a beam equation on a nonlinear elastic foundation," Mathematical and Computer Modelling, vol. 39, no. 11-12, pp. 1195-1201, 2004.

[3] G. Bonanno and B. Di Bella, "A boundary value problem for fourth-order elastic beam equations," Journal of Mathematical Analysis and Applications, vol. 343, no. 2, pp. 1166-1176, 2008.

[4] G. Bonanno and B. Di Bella, "Infinitely many solutions for a fourth-order elastic beam equation," Nonlinear Differential Equations and Applications, vol. 18, no. 3, pp. 357-368, 2011.

[5] G. Han and Z. Xu, "Multiple solutions of some nonlinear fourthorder beam equations," Nonlinear Analysis: Theory, Methods and Applications, vol. 68, no. 12, pp. 3646-3656, 2008.

[6] X.-L. Liu and W.-T. Li, "Existence and multiplicity of solutions for fourth-order boundary value problems with parameters," Journal of Mathematical Analysis and Applications, vol. 327, no. 1, pp. 362-375, 2007.

[7] T. F. Ma, "Existence results and numerical solutions for a beam equation with nonlinear boundary conditions," Applied Numerical Mathematics, vol. 47, no. 2, pp. 189-196, 2003. 
[8] L. Yang, H. Chen, and X. Yang, "The multiplicity of solutions for fourth-order equations generated from a boundary condition," Applied Mathematics Letters, vol. 24, no. 9, pp. 1599-1603, 2011.

[9] F. Li and J. Sun, "Infinitely many homoclinic solutions for a nonperiodic fourth-order differential equation without (AR)condition," Applied Mathematics and Compution. In press.

[10] H. Chen and J. Sun, "An application of variational method to second-order impulsive differential equation on the half-line," Applied Mathematics and Computation, vol. 217, no. 5, pp. $1863-$ 1869, 2010.

[11] J. R. Graef, S. Heidarkhani, and L. Kong, "A variational approach to a Kirchhoff-type problem involving two parameters," Results in Mathematics, vol. 63, pp. 877-889, 2013.

[12] B. Ricceri, “On an elliptic Kirchhoff-type problem depending on two parameters," Journal of Global Optimization, vol. 46, no. 4, pp. 543-549, 2010.

[13] J. Sun, H. Chen, J. J. Nieto, and M. Otero-Novoa, "The multiplicity of solutions for perturbed second-order Hamiltonian systems with impulsive effects," Nonlinear Analysis: Theory, Methods and Applications, vol. 72, no. 12, pp. 4575-4586, 2010.

[14] B. Ricceri, "A further three critical points theorem," Nonlinear Analysis, Theory, Methods and Applications, vol. 71, no. 9, pp. 4151-4157, 2009.

[15] B. Ricceri, "A three critical points theorem revisited," Nonlinear Analysis: Theory, Methods and Applications, vol. 70, no. 9, pp. 3084-3089, 2009.

[16] B. Ricceri, "Existence of three solutions for a class of elliptic eigenvalue problems," Mathematical and Computer Modelling, vol. 32, no. 11-13, pp. 1485-1494, 2000.

[17] E. Zeidler, Nonlinear Functional Analysis and Its Applications, Springer, Berlin, Germany, 1990. 


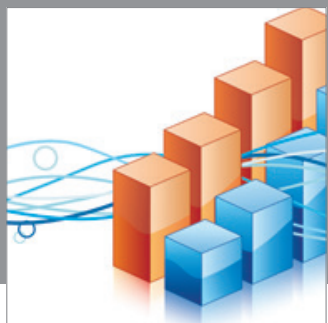

Advances in

Operations Research

mansans

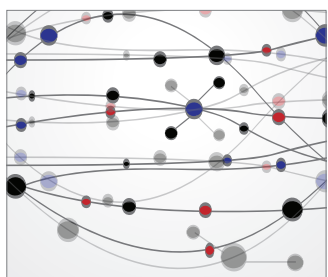

The Scientific World Journal
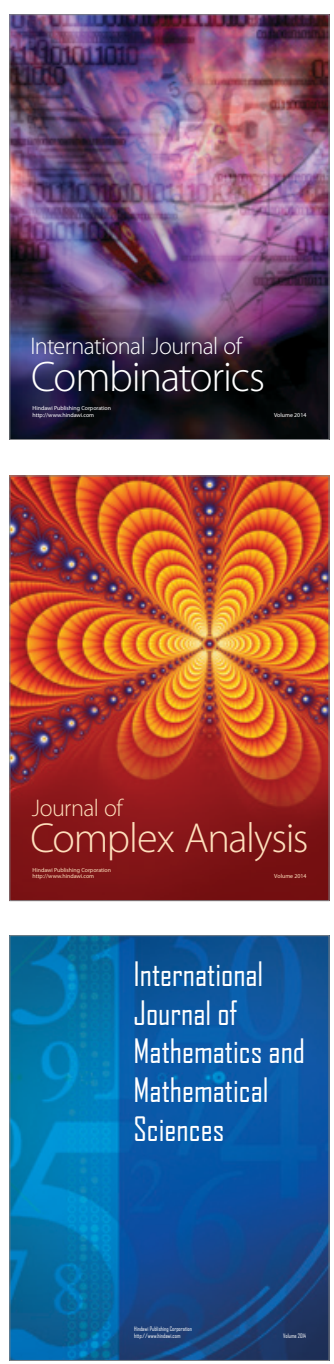
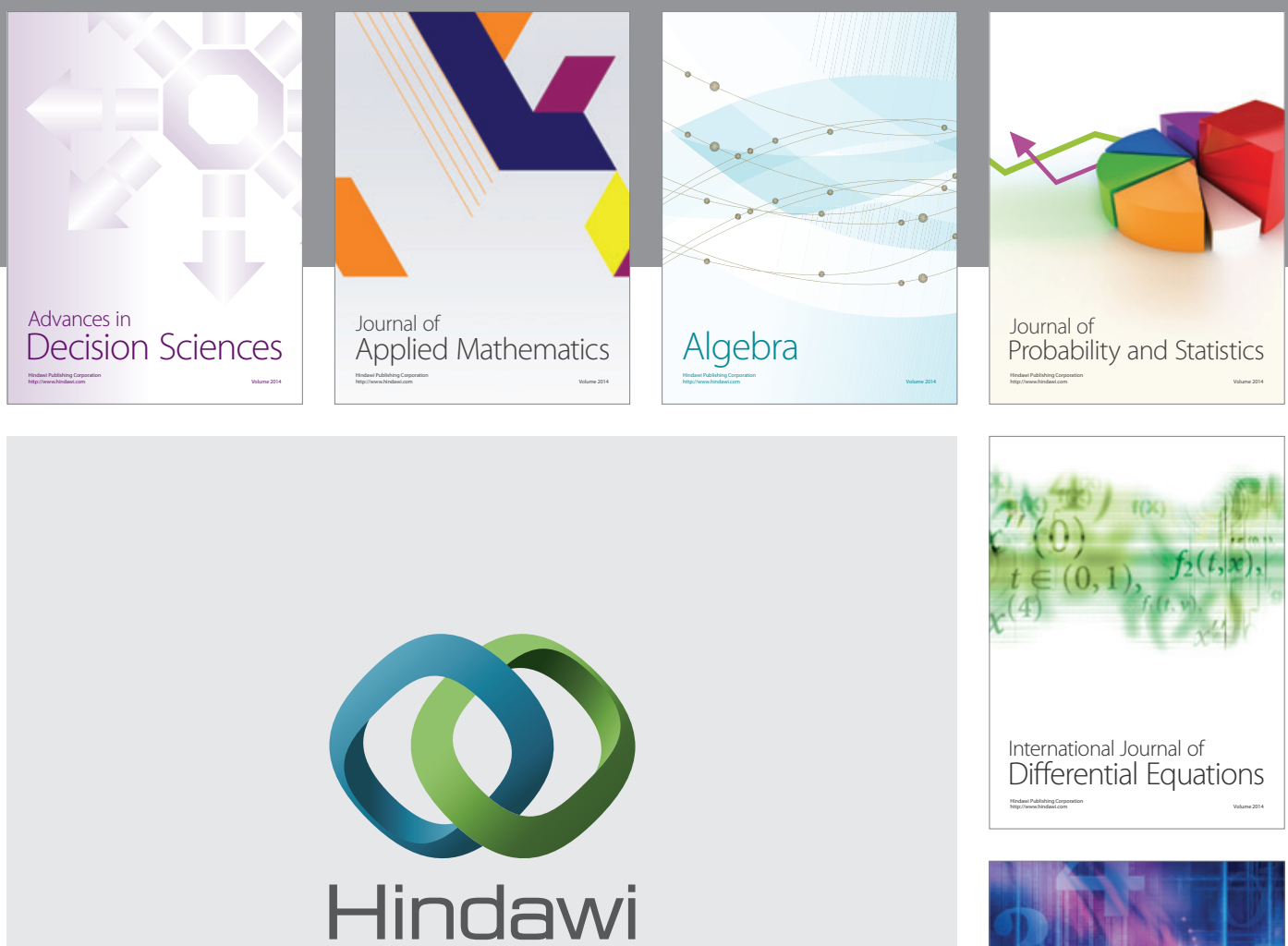

Submit your manuscripts at http://www.hindawi.com
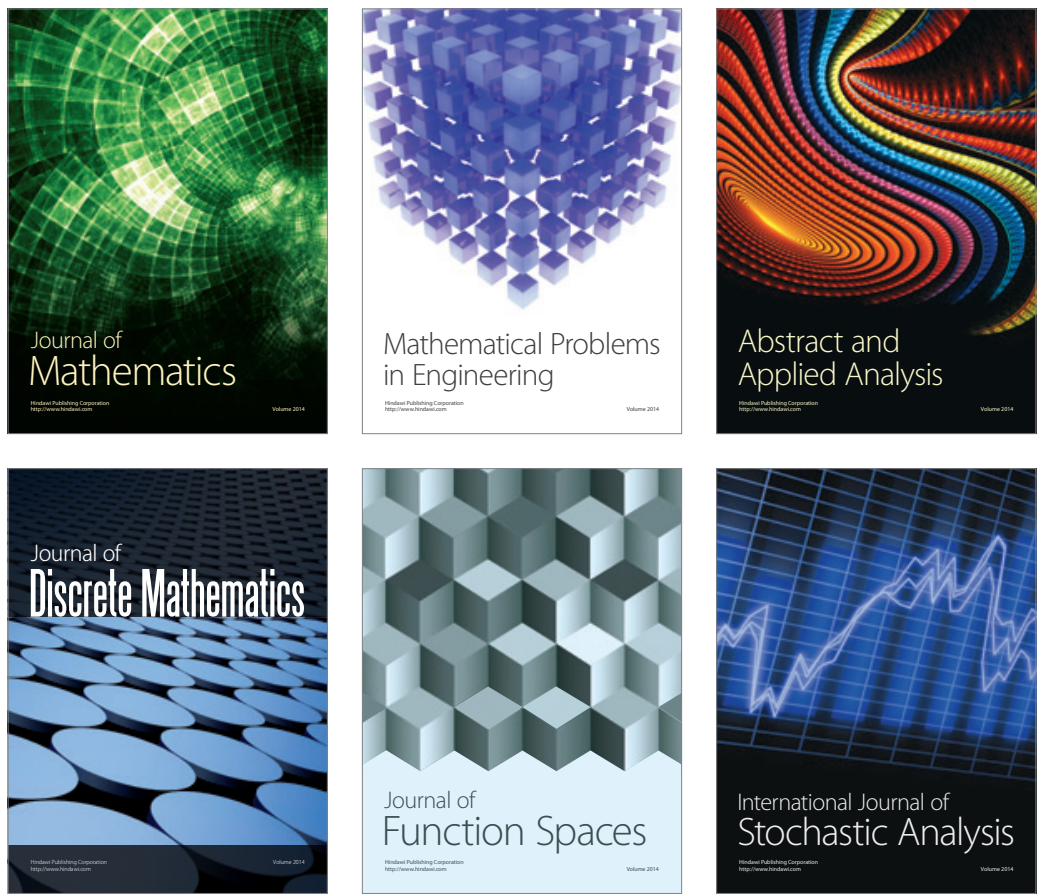

Journal of

Function Spaces

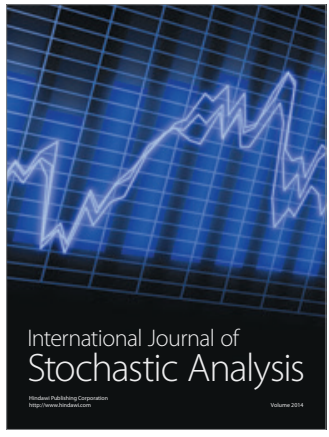

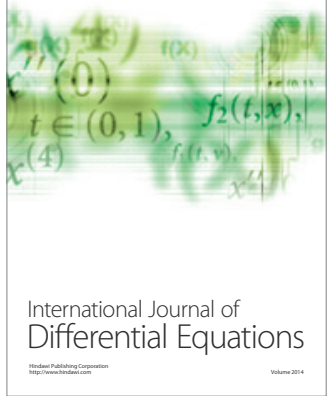
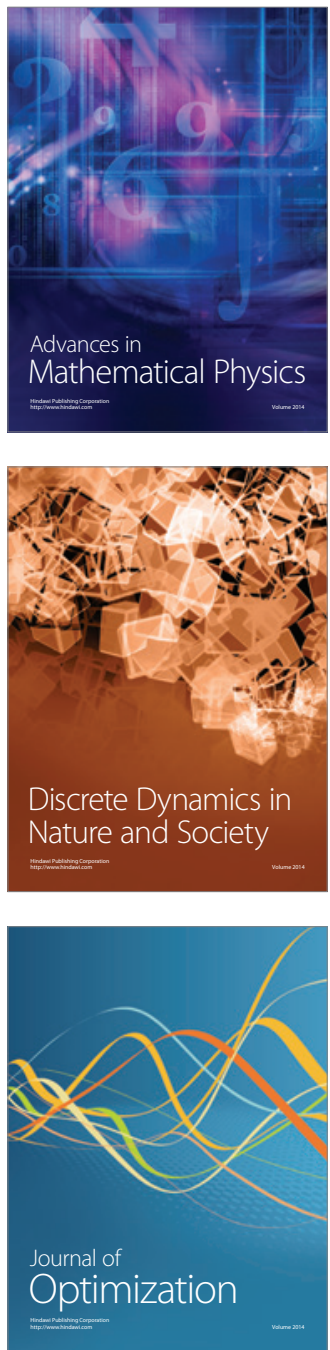